\title{
ISENTROPIC COMPRESSION EXPERIMENTS ON THE SANDIA Z ACCELERATOR
}

Clint A. Hall

Sandia National Laboratories, Albuquerque, NM $87185^{1}$

A long-standing goal of the equation of state (EOS) community has been the

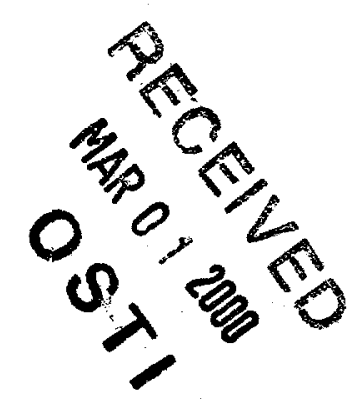
development of a loading capability for direct measurement of material properties along an isentrope. Previous efforts on smooth bore launchers have been somewhat successful, but quite difficult to accurately reproduce, had pressure limitations, or tended to be a series of small shocks as opposed to a smoothly increasing pressure load. A technique has recently been developed on the Sandia National Laboratories $\mathrm{Z}$ accelerator which makes use of the high current densities and magnetic fields available to produce nearly isentropic compression of samples that are approximately $1 \mathrm{~mm}$ in thickness over approximately $120 \mathrm{~ns}$. Velocity interferometry is used to measure the rear surface motion of these samples. The resulting time resolved velocity profiles from multiple sample thicknesses provide information about mechanical response under isentropic loading conditions and phase transition kinetics. Feasibility experiments have been performed to pressures of approximately $130 \mathrm{kbar}$ in copper and $300 \mathrm{kbar}$ in iron with effects of the $\alpha$ $\varepsilon$ phase change kinetics in iron clearly observed. Work is in progress to achieve $1-2 \%$ accuracy in P-v space along an isentrope, provide uniaxial strain, and to eliminate magnetic field and current diffusion within the sample of interest.

\footnotetext{
'Sandia is a multiprogram laboratory operated by Sandia Corporation, a Lockheed Martin Company, for the United States Department of Energy under Contract DE-AC04-94AL8500.
} 


\section{DISCLAIMER}

This report was prepared as an account of work sponsored by an agency of the United States Government. Neither the United States Government nor any agency thereof, nor any of their employees, make any warranty, express or implied, or assumes any legal liabiiity or responsibility for the accuracy, completeness, or usefulness of any information, apparatus, product, or process disclosed, or represents that its use would not infringe privately owned rights. Reference herein to any specific commercial product, process, or service by trade name, trademark, manufacturer, or otherwise does not necessarily constitute or imply its endorsement, recommendation, or favoring by the United States Government or any agency thereof. The views and opinions of authors expressed herein do not necessarily state or reflect those of the United States Government or any agency thereof. 


\section{DISCLAIMER}

Portions of this document may be illegible in electronic image products. Images are produced from the best available original document. 


\section{Introduction}

The high pressure equation-of-state (EOS) of materials is typically determined by subjecting the material to a steady, fully developed shock wave and making measurements of shock speed and mass velocity. Applying the Hugoniot jump conditions which describe conservation of mass, momentum and energy across this abrupt discontinuity in stress and density within the material allow the determination of the material's Hugoniot curve on its EOS surface. The Hugoniot curve, however, is irreversible and only represents a locus of end states achieved through steady-state shock compression, so many experiments are usually performed to identify the Hugoniot curve.

Hugoniot techniques have been useful for generating high-pressure EOS properties, but there are many applications where loading paths lie much closer to a material isentrope than the Hugoniot curve. An isentrope is a reversible path of constant entropy on an equation-of-state surface where the entire change in a material's internal energy is due to Pdv work. Techniques for generating isentropic loading curves using ramp generators ${ }^{1}$ and graded density impactors ${ }^{2}$ on launchers have been used successfully in past material property studies, but have pressure and reproducibility limitations. Laser-driven isentropic loading has also been tried, but it is difficult to couple laser energy into the target over long time scales (approximately $200 \mathrm{~ns}$ ) which are desirable for this technique.

A new capability for producing isentropic compression using fast pulsed power as the energy source is currently being developed on the $\mathrm{Z}$ Accelerator ${ }^{3}$. The $\mathrm{Z}$ accelerator is a low inductance pulsed power generator capable of capacitively storing $11.6 \mathrm{MJ}$ of 
electrical energy which, when discharged, creates currents of $22 \mathrm{MA}$. When the machine fires, this current is delivered to the central target by 36 transmission lines arranged like spokes on a wheel and insulated by water. The resulting electrical pulse achieves powers as high as 50 terawatts at the vacuum interface of the target chamber. This drive source offers many advantages over previous experimental techniques for isentropic compression. These advantages include a smoothly increasing loading profile without any initial low-level shocks, the ability to investigate many samples experiencing "identical" loading, and a loading profile that can be easily tailored to meet experimental demands. It is important to note that this process is close, but not perfectly isentropic. It is shockless and adiabatic due to experimental timescales, but is not reversible due to the presence of elastic-plastic behavoir, dissipative dynamic viscosity effects, and the presence of any non-equilibrium phase transistions. An overview of this approach to isentropic loading, along with recent pressure-volume results to approximately $200 \mathrm{kbar}$ in copper, will be discussed. Wave profiles demonstrating the evolution of the $\alpha-\varepsilon$ phase transition in iron will also be presented.

\section{Experimental Technique}

The principle that allows pulsed power to apply shockless loading to EOS samples is shown in Figure 1. A short circuit is created between the anode and cathode in the $Z$ Accelerator that results in a current flow on the inner surface of each component. The interaction between the current density and magnetic field produced in the insulating gap produces a pressure that is applied to the inner surface of the sample. Since magnetic pressure scales with $B^{2}$, and $B$ is $\left(\mu_{0}\right) J$ where $J$ is current density, or $I / W$, the magnitude of this loading is given by 


$$
\mathrm{P}=\left(\mathrm{I}^{2} \mu_{0}\right) /\left(2 \mathrm{~W}^{2}\right)
$$

where $P$ is the load applied to the sample in GPa, $I$ is the current in amps, $W$ is the width (or perimeter) of the conductor at the sample location on which current flows, in meters, $\mathrm{B}$ is the magnetic field strength in tesla, and $\mu_{0}$ is the magnetic permeability of free space. As can be seen, the pressure will follow the risetime of the applied current profile, but the magnitude of the loading is easily influenced by modifying the input current, or by decreasing the current carrying surface at the sample location.

The three different experimental configurations that have been used on the $\mathrm{Z}$ Accelerator to isentropically load samples are shown in Figure 2. The geometry in Figure 2a was used with iron samples in the form of cylindrical disks pressed into place simply for technique demonstration. In this configuration the current density is not constant due to converging current (a decreasing $\mathrm{W}$ term as a function of anode radius for a given I) that causes a pressure gradient across the sample diameter, thus limiting its use for highly accurate EOS data. Figure $2 \mathrm{~b}$ represents the configuration used to gather data on copper samples. It is a preferable geometry because the pressure gradient is limited to the effect from a local, nonsymmetrical gap between the anode and cathode caused by a $6 \mathrm{~mm}$ chord placed in the wall of a $40 \mathrm{~mm}$ diameter tube. Samples were machined in the form of a cup and pressed into place with a 0.0005 " interference fit between sample and cathode to provide current contact and eliminate the need for adhesives in this portion of the machine. Figure $2 \mathrm{c}$ shows the first attempt at designing a load to obtain accurate isentropic data on Z. As seen, the anode and cathode consist of flat panels that maintain a symmetric current density, and therefore pressure, over the samples during the loading process. 2-D calculations by Reisman using $\mathrm{Trac} \mathrm{II}^{4}$ indicate that symmetry of loading 
across each sample face is 0.03 percent in the horizontal direction. Calculations performed at Sandia National Laboratories using Quicksilver indicate a 0.7 percent difference in pressure between the upper and lower samples. This apparent symmetry suggests that data can be compared between samples to obtain EOS information.

Time resolved mass velocity data is recorded from the rear surface of each sample using velocity interferometry, $\operatorname{VISAR}^{5}$, coupled through fiber optics to provide the information necessary for inferring stress and specific volume. Current profiles are measured with Bdot probes at several radial positions on the current transmisson lines.

\section{Analysis and Results}

Typical studies of material properties using shock compression are performed on precision launchers to create flat plate impacts. Under these conditions, a fully developed shock, which remains constant in amplitude and risetime over the experimental timeframe, is generated. A series of equations, commonly known as the Hugoniot jump conditions ${ }^{6}$, that describe conservation of mass, momentum and energy across the shock front are used to infer the material properties of interest. The isentropic loading technique, however, investigates the compressive wave evolution before it becomes fully developed by measuring the risetime of the mass velocity profile at different material depths to allow calculation of Lagrangian wave speed, $\mathrm{C}_{\mathrm{L}}$, as a function of mechanical stress. This measurement, along with the differential form of the conservation conditions shown below, allow the path dependent stress and specific volume to be calculated from initial conditions to their final compressed states.

$$
\begin{aligned}
& d \sigma=\left(1 / v_{0}\right) C_{L} d u_{P} \\
& d v=-v_{0}\left(d u_{P} / C_{L}\right)
\end{aligned}
$$




$$
\mathrm{dE}=-\mathrm{Pdv}
$$

These equations, however, are only valid if the initial state of the material is known, and simple waves with no strain rate dependence are observed. A concern of the magnetically loaded isentropic technique is that the magnetic field might diffuse within the material at a faster rate than the hydrodynamic wave that we are measuring. This would result in a preconditioning of the sample material and an unknown initial state. Calculations by Reisamn using Trac $\mathrm{II}^{4}$ and Rosenthal using Mach $\mathrm{II}^{7}$ indicate that the hydrodynamic response travels ahead of the current diffusion in both copper and iron at these stress levels and current-time histories. Calculations for copper at approximately 280 kbar are shown in Figure 3.

The rear surface velocity profiles and input current profile for $Z$ shot 452 using the experimental configuration shown in Fibure $2 \mathrm{~b}$ are shown in Figure 4. As expected, the risetime of the loading wave measured by VISAR at the rear surface of each sample decreases as the wave travels through the $0.5 \mathrm{~mm}$ and $0.8 \mathrm{~mm}$ thick copper samples. A portion of the wave has almost developed into a shock front at the $0.8 \mathrm{~mm}$ thickness, but does still have a finite, measurable risetime so can be used with equations 2 and 3 for isentropic loading analysis.

Before the Lagrangian sound speed as a function of mass velocity can be calculated, the wave profiles must be time correlated and a correction factor applied to account for wave interactions at the free surface of each sample ${ }^{8}$. Asay has discussed the need for this correction factor in previous isentropic loading experiments on guns using ramp generators ${ }^{1}$. Since the wave interactions at a free surface experiencing timedependent loading are quite complex, they require an iterative analysis to converge on the 
final answer. This was accomplished in the present study by calculating the wave profiles at each free sample surface using $\mathrm{Trac} \mathrm{II}^{4}$, applying $\mathrm{u}_{\mathrm{p}} / 2$ approximations (which assume that the insitu particle velocity is exactly one half the measured free surface velocity), and comparing the result to wave profile calculations at the same sample thicknesses within infinitely thick samples of copper. The result, shown in Figure 5, indicated that a linearly increasing correction from 0 to approximately $2 \%$ be applied to the time interval between the two wave profiles. Once applied, increments of $0.05 \mathrm{~km} / \mathrm{s}$ in mass velocity were chosen to provide adequate resolution for defining a P-v curve in copper. With digitizing speed limited to $200 \mathrm{ps} /$ point and the approximately $1 \mathrm{~ns}$ risetime seen in a portion of the $0.8 \mathrm{~mm}$ thick sample, several of the transit times had to be interpolated for sound speed determination. Stress was calculated using equation 2 and a curve plotting sound speed as a function of pressure generated. This result, compared to Hugoniot data from Kinslow ${ }^{9}$, is shown in Figure 6. With the local wave speed as a function of pressure determined, the pressure-volume curve could then be calculated using equation (3). Figure 7 shows the result again compared to Hugoniot data from several sources ${ }^{[9,10,11,12]}$. As can be seen, one isentropic loading experiment defines a large portion of the P-v material response, whereas conventional Hugoniot techniques define only one point per experiment. This represents a major advantage for this technique.

Comparisons between Hugoniot and isentropic pressures are valid in this stress regime for many materials, including copper. The difference between the pressure along the Hugoniot, $\mathrm{P}_{\mathrm{H}}$, and the pressure along the isentrope, $\mathrm{P}_{\mathrm{S}}$, is proportional to volumetric strain cubed as shown in equation 5 , where $B_{0}$ is the bulk modulus, $B_{0}$ ' is the first 
derivative of the bulk modulus with respect to pressure, $\gamma_{0}$ is the Gruneisen parameter, and $\varepsilon$ is the volumetric strain. For $200 \mathrm{kbar}$ in copper, the difference between the two pressures is calculated to be less than $1 \%$.

$$
\mathrm{P}_{\mathrm{H}}(\mathrm{v}) \approx \mathrm{P}_{\mathrm{S}}(\mathrm{v})+\gamma \varepsilon^{3}\left(\mathrm{~B}_{0}\left(\mathrm{~B}_{0}{ }^{\prime}+1\right)\right) / 12
$$

There are several sources of error that can influence the P-v results obtained using ICE techniques. Most obvious is the ability to accurately time correlate the measured velocity profiles from which local wave speed is calculated. For experiment Z452, it was possible to perform this operation to within $0.5 \mathrm{~ns}$. With transient times of approximately $70 \mathrm{~ns}$ between velocity profiles at a given pressure state, errors of about $1 \%$ are introduced into calculations of $\mathrm{C}_{\mathrm{L}}$ and will carry forward into the $\mathrm{P}-\mathrm{v}$ results. The inherent accuracy of the VISAR itself can also be a source of error. With the high signal to noise ratio for these velocity profiles, the uncertainty is $2 \%$ of the fringe constant which was set at $0.81 \mathrm{~km} / \mathrm{sec} /$ fringe on this experiment. This, therefore, leads to an associated error of approximately $1 \%$ as well.

Another potential source for error is the fiber-optic probe arrangement used to collect the Doppler-shifted light from the rear surface of the sample. In this probe, a 200 micron core fiber used to send light from a doubled Yag laser is placed adjacent to a 200 micron core fiber which collects light reflected from the sample surface and sends it back to the interferometer. With the probe positioned $0.5 \mathrm{~mm}$ from the rear sample surface, and the numerical aperature of the fiber at 0.22 , it can be shown that a cosine error ${ }^{8}$ of approximately $2 \%$ is introduced into the velocity measurement due to collection of Doppler shifted light which is not normal to the moving surface. The result is a velocity 
profile that is slightly lower in amplitude than it should be. The data was corrected for this effect by applying a $(1 / \cos )$ correction factor ${ }^{13}$ to the VISAR fringe constant.

A more detailed discussion of error sources and effects will be published for isentropic loading using this technique ${ }^{8}$ and quantifies the total error to be approximately $3 \%$ on the final values of pressure and specific volume shown in Figure 7. Efforts are currently underway to improve the accuracy of these measurements. Use of a Line ORVIS ${ }^{14}$, which records multiple velocity profiles with spatial resolution on a sample surface, combined with stepped samples should allow timing accuracy sufficient for 1$2 \% \mathrm{P}-\mathrm{v}$ data by recording velocity profiles from both steps on a single streak camera. In addition, the system being developed on $Z$ will be lens coupled which will eliminate the need for the $1 /$ cosine correction factor to the resulting velocity profiles.

In addition to providing a method for determining the P-v response of materials along their isentrope, this method of loading allows the kinetics of phase transitions to be investigated more easily than when more traditional shock methods are used. To demonstrate this effect, the $\mathrm{Z}$ accelerator was used to isentropically load Armco iron. Iron has a well-established solid-solid phase transition from the bcc $(\alpha)$ to the hcp ( $\varepsilon$ ). phase that occurs at approximately $130 \mathrm{kbar}$ and has been investigated by Barker using wave profile techniques on smooth bore launchers ${ }^{15}$. It is difficult to study phase transitions subjected to fully developed shocks because Rayleigh Lines define the path followed during shock compression. The first path with slope equal to $\rho_{0} U_{s}$ connects the initial state to the pressure where the phase transition occurs. A second path, or Rayleigh line, connects the phase transition to the final compressed state with a different slope and thus, different wave speed. This leads to a "fully developed" two-wave structure with a 
definite discontinuity indicating the existence of the phase transition as shown in Figure 8. Kinetics of phase transitions can be inferred from these types of wave profiles by investigating the time required for the amplitude of the wave discontinuity to become steady state, but is difficult due to the small pressure changes which occur as the superheated bcc phase obtains equilibrium. Barker has also demonstrated that the transition in iron is not instantaneous under shock loading, but instead has a transition time of approximately $40 \mathrm{~ns}$.

The experimental configuration shown in Figure 2a was used on the $Z$ accelerator to demonstrate the potential for investigating phase transitions in two samples of iron under isentropic loading. Each of the samples was a right circular cylinder, $3 \mathrm{~mm}$ in diameter with thicknesses of $0.5 \mathrm{~mm}$ and $0.8 \mathrm{~mm}$ respectively. As with the copper samples, they were pressed in place to eliminate the need for glue where current densities are so high. Both sample surfaces were highly polished and parallel to within approximately 10 microns. A VISAR was fiberoptically coupled to the rear of each sample to monitor surface motion.

An inherent problem exists with this experimental configuration, however, that limits its use for obtaining highly accurate information. The current flow in the anode where samples are placed is convergent. Because the current density is higher at the sample edge closer to $r=0$, the axis of symmetry, the pressure is higher at that location as well. This can be shown from equation 1 by substituting $2 \pi \mathrm{r}$ for $\mathrm{W}$. The consequences for this effect are a pressure gradient of approximately $7 \%$ across the portion of the sample being illuminated by the VISAR probe. While not adequate for accurate EOS 
measurements, it is still adequate to demonstrate the technique as a viable method for investigating phase transitions.

The two free surface velocity profiles recorded during this experiment are shown in Figure 9. The elastic precursor is clearly seen in the lower pressure region of the two profiles. Calculation of the elastic wave speed from these profiles results in a velocity of $6.24 \mathrm{~km} / \mathrm{s}$ which is in good agreement with reported values of $6.36 \mathrm{~km} / \mathrm{s}$ obtained by Barker $^{15}$. Of greater interest though, is the region where the phase transition is occurring. Since the isentropic loading technique actually follows the P-v response of the material under investigation, the actual evolution of the phase transition can be observed. The velocity profile for the $0.5 \mathrm{~mm}$ thick iron sample in Figure 9 has an arrow that indicates the onset of the phase transition before arrival of the wave traveling in the hcp phase material. The $0.8 \mathrm{~mm}$ thick profile has a more fully evolved transition that more closely resembles results typically seen in plate impact experiments.

From these profiles, the kinetics of the transition can be estimated. Hayes has modified a one dimensional wave propagation code (WONDY) to include the effects of kinetics in phase transformations. The model used employs $\alpha$ and $\varepsilon$ equations of state from Andrews ${ }^{16}$ and the pressure and temperature at each time and position are advanced using the finite difference form of the equations in (6).

$$
\left.\left.\left(\begin{array}{l}
\dot{p} \\
\dot{T}
\end{array}\right)=\left(\begin{array}{lll}
a_{S, x}^{2} & \frac{K_{s, x}}{V C_{p, x}}\left[\left(\frac{\partial E}{\partial T}\right)_{p, x}\right. & \Delta V-\left(\frac{\partial V}{\partial T}\right)_{p, x} \Delta E
\end{array}\right]\right)\left(\begin{array}{ll}
\dot{\rho} \\
\frac{\gamma_{x} T}{\rho}-\frac{K_{s, x}}{V^{2} C_{p, x}}\left[\left(\frac{\partial E}{\partial \rho}\right)_{T, x}\right. & \Delta V-\left(\frac{\partial V}{\partial p}\right)_{T, x} \Delta E
\end{array}\right]\right)(\dot{x})
$$


Equation (6) ${ }^{17}$ evaluates all thermodynamic quantities at fixed mass fractions of the phases (x), and assumes that the mixture of $\alpha$ and $\varepsilon$ phases is locally at uniform pressure and temperature as it evolves to a state of equilibrium. All other terms have their conventional meanings ${ }^{18}$. Attempts to model the velocity profiles obtained on $Z$ assumed constant resistivity within the iron. An additional term was added into the magnetic diffusion equation to account for flux compression in materials with finite strain rates, and gradients in the sum of the mechanical and magnetic pressure are used in the momentum equation to calculate the effects of the shockless loading. Internal energy increases occur because of mechnaincal work (Pdv), joule heating, and the work done against the Lorentz force. The rate in this kinetics model is not constant and is allowed to vary during the calculation. The rate is observed to vary from 0 at $130 \mathrm{kbar}$ (onset of the phase transition) to $50 \mu \mathrm{s}^{-1}$ when the peak stress is reached at the top of the ramp wave. The average rate is therefore $25 \mu \mathrm{s}^{-1}$, which correlates to a phase change time of approximately $40 \mathrm{~ns}$, in agreement with the empirical model of Barker and Hollenbach ${ }^{15}$.

\section{Summary}

In summary, a new method to shocklessly load materials has been developed using pulsed power as the energy source. The technique takes advantage of the high current densities and magnetic fields available on the Sandia $\mathrm{Z}$ accelerator to provide smoothly increasing pressure pulses of 100-200 ns to achieve pressures that will approach $1 \mathrm{Mbar}$ in future studies. Shockless, adiabatic loading of this type is nearly isentropic, so material response along an isentrope can be experimentally determined by recording the evolution of a wave profile at multiple sample thicknesses using VISAR, calculating the 
local sound speed, and applying a differential form of the Hugoniot jump conditions. This technique extends the pressure range and accuracy possible using previously developed plate impact methods that employed graded density impactors and ramp generating materials. Recent experiments have been performed on copper to obtain an isentropic pressure-volume response to approximately $160 \mathrm{kbar}$ which is compared to published Hugoniot data. Armco iron was also investigated to demonstrate the possibilities of studying phase transitions using isentropic loading. Efforts are currently underway to improve the accuracy of the P-v data to approximately 1-2\% through load design, improved instrumentation, and refinements to data analysis techniques.

\section{Acknowledgements}

The author wishes to acknowledge the efforts of all those that have contributed to the development of ICE loading on $\mathrm{Z}$ that is discussed only briefly in this review. Most notably, James Asay who's past work in this area, technical expertise, and vision for the future of this technique have had a major impact on its development. Others that have made significant contributions include W.A. Stygar, R.B. Spielman, M.D. Knudson, D. Reisman, A. Toor, R. Cauble, D. B. Hayes, S.E. Rosenthal, and M.A. Bernard. 
Figure 1: a schematical representation of the current path and resultant magnetic field created between the anode and cathode of the $Z$ accelerator which loads the sample with a smoothly increasing, isentropic pressure pulse.

Figure 2: Sectioned views of the three different experimental configurations that have been used to gather isentropic data on the $\mathrm{Z}$ accelerator. $2 \mathrm{a}$ was used with iron samples, $2 \mathrm{~b}$ was used with copper samples, and $2 \mathrm{c}$ represents the first attempt at designing a load to produce more controlled, planar loading for this technique.

Figure 3: Calculations using the MHD code Trac II which demonstrate the hydrodynamic waves position in time relative to the predicted magnetic field diffusion. Curves were generated for both constant and temperature dependent resistivity.

Figure 4: The recorded free surface velocity profiles and input current profile for shot Z452. This experiment used copper samples mounted in the anode-cathode arrangement depicted in Figure 2b.

Figure 5: A Trac II calculation showing the free surface correction applied to the Z452 data prior to determination of local sound speeds. The calculation compared the free surface velocity with a $u_{p} / 2$ approximation to an insitu velocity profile in an infinately thick sample of copper at the same sample thickness.

Figure 6: An experimentally determined sound speed as a function of pressure curve for the copper data of shot $Z 452$ after application of the calculated free surface correction factor. The results are compared to Hugoniot data from Kinslow. 
Figure 7: An experimentally determined pressure-volume curve for copper to approximately $160 \mathrm{kbar}$ using the isentropic compression technique on $\mathrm{Z}$. Results are compared to published Hugoniot data.

Figure 8: A "conceptual" representation showing the shock path along Rayleigh lines from ambient conditions to a phase transition, and phase transition to the final compressed state. A typical velocity profile that would result from this type of shock loading is also shown.

Figure 9: Velocity profiles obtained on the free surface of 0.6 and $0.9 \mathrm{~mm}$ thick iron samples subjected to isentropic loading on the $\mathrm{Z}$ accelerator. The kinetics of the phase transition can be clearly seen as it evolves toward a state of equilibrium. 


\section{References}

1. Asay, J.R. and L.C. Chhabildas, Some New Developments in Shock Wave Research, High Pressure Science and Technology, Proceedings of the $7^{\text {th }}$ Association International for Research and Advancement of High Pressure Science and Technology (AIRAPT), Villetaneuse, France, ed. By B. Vodar and P. Marteau, Pergamon Press, Vol 2, 958 - 965, (1980)

2. Chhabildas, L.C. and J. R. Asay, Dynamic Yield Strength and Spall Strength Measurements Under Quasi-Isentropic Loading, Shock Waves and High-Strain Rate Phenomena in Metals, Proceedings of the International Conference on the Material Effects of Shock-Wave and High-Strain-Rate Phenomena, 1990 , edited by M.A. Meyers and L.E. Murr, and K.P. Staudhammer. Marcel Dekker, Inc., pp 947-955.

3. Matzen, M.K., Z pinches as intense X-ray sources for high-energy density physics applications, Physics of Plasmas, 4 (5), 1519 - 1527 (1996)

4. Reisman, D.B., A. Toor, R. Cauble, C.A. Hall, J.R. Asay, M.D. Knudson, Magnetically Driven Isentropic Compression Experiments on the Z Accelerator, Journal of Applied Physics, to be submitted, 2000

5. Barker, L.M. and R.E. Hollenbach, Laser interferometer for measuring high velocities of any reflecting surface, Journal of Applied Physics, Vol. 43 no. 11, pg 4669-4675, (1972)

6. Asay, J.R. and M. Shahinpoor, editors, High Pressure Shock Compression of Solids, Springer-Verlag New York, Inc., 1993

7. Rosenthal, S.E., M.P. Desjarlais, R.B. Spielman, W.A. Stygar, J.R. Asay, M.R. Douglas, C.A. hall, M.H. Frese, R.L. Morse, and D.B. Reisman, MHD Modeling of 
Conductors at Ultra-High Current Density, submitted to Institute of Electrical and Electronics Engineers (IEEE) Transactions on Plasma Science, 1999

8. Hall, C.A., J.R. Asay, W.A. Stygar, et al., Use of Fast Pulsed power techniques for Isentropic Compression Experiments, Review of Scientific Instruments, to be submitted, 2000

9. Kinslow, R., High-Velocity Impact Phenomena, Academic Press, 111 Fifth Avenue, NY, NY 10003, (1970)

10. Chhabildas, L.C., and J.R. Asay, Time-Resolved Wave Profile Measurements in Copper to Megabar Pressures, High Pressure in Research and Industry, Proceedings of the $8^{\text {th }}$ Association International for Research and Advancement of High Pressure Science and Technology (AIRAPT), University of Uppsala, Sweden, ed. by C.M. Backman, T. Johannisson, and L. Tegner, Vol 1, 183 - 189, (1981)

11. Marsh, S.P., editor, LASL Shock Hugoniot Data, University of California Press, Berkeley, Ca., 1980

12. Walsh, J.M., M.H. Rice, R.G. McQueen, F.L. Yarger, Shock-Wave Compressions of Twenty-Seven Metals. Equation of State of Metals, Physical Review, vol. 108 no. 2, $196-216(1957)$

13. Chhabildas, L.C., Dynamic Shear-Wave Velocity Measurements Using Interferometric Techniques, Proceedings of the International Congress on Instrumentation in Aerospace Simulation Facilities, publication no. 81CH1712-9, Institute of Electrical and Electronics Engineers (IEEE), Dayton, Ohio, 1981

14. Trott, W.M., M.D. Knudson, L.C. Chhabildas, J.R. Asay, Measurements of Spatially Resolved Velocity Variations in Shock Compressed Heterogeneous Materials Using a 
Line Imaging Velocity Interferometer, proceedings of the American Physical Society topical group on Shock Compression of Condensed Materials, Snowbird, Utah, July 1999

15. Barker, L.M. and R.E. Hollenbach, Shock wave study of the $\alpha \leftrightarrow \varepsilon$ phase transition in iron, Journal of Applied Physics, vol. 45 no. 11, pp $4872-4887$ (1974)

16. Andrews, D.J., Equation of State of the Alpha and Epsilon Phases of Iron, Journal of Physics in Chemical Solids, 34, Pergamon Press, 825-840 (1973)

17. Asay, J.R., C.A. Hall, K.G. Holland, M.A. Bernard, W.A. Stygar, R.B. Spielman, S.E. Rosenthal, D.H. McDaniel, and D.B. Hayes, Isentropic Compression of Iron with the Z Accelerator, proceedings of the American Physical Society topical group on Shock Compression of Condensed Materials, Snowbird, Utah, July 1999

18. Hayes, D.B., Wave propagation in a condensed medium with $\mathrm{N}$ transforming phases: application to Solid-I-Solid-II-liquid bismuth, Journal of Applied Physics, vol. 46, $3438(1975)$ 


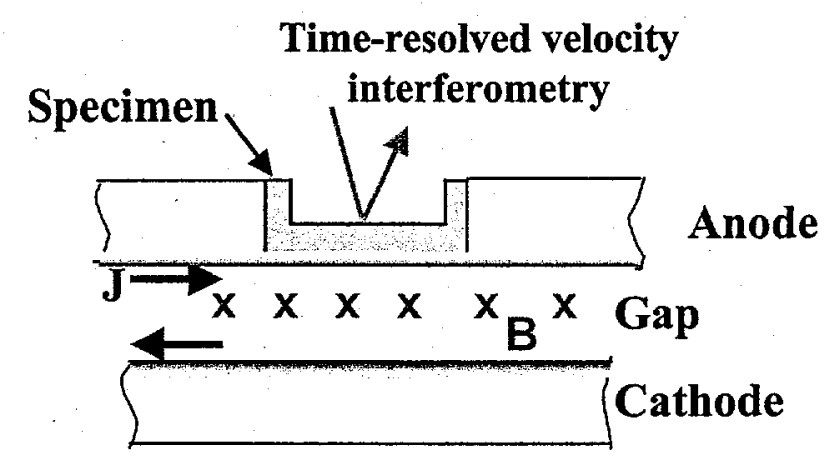

Figure 1 


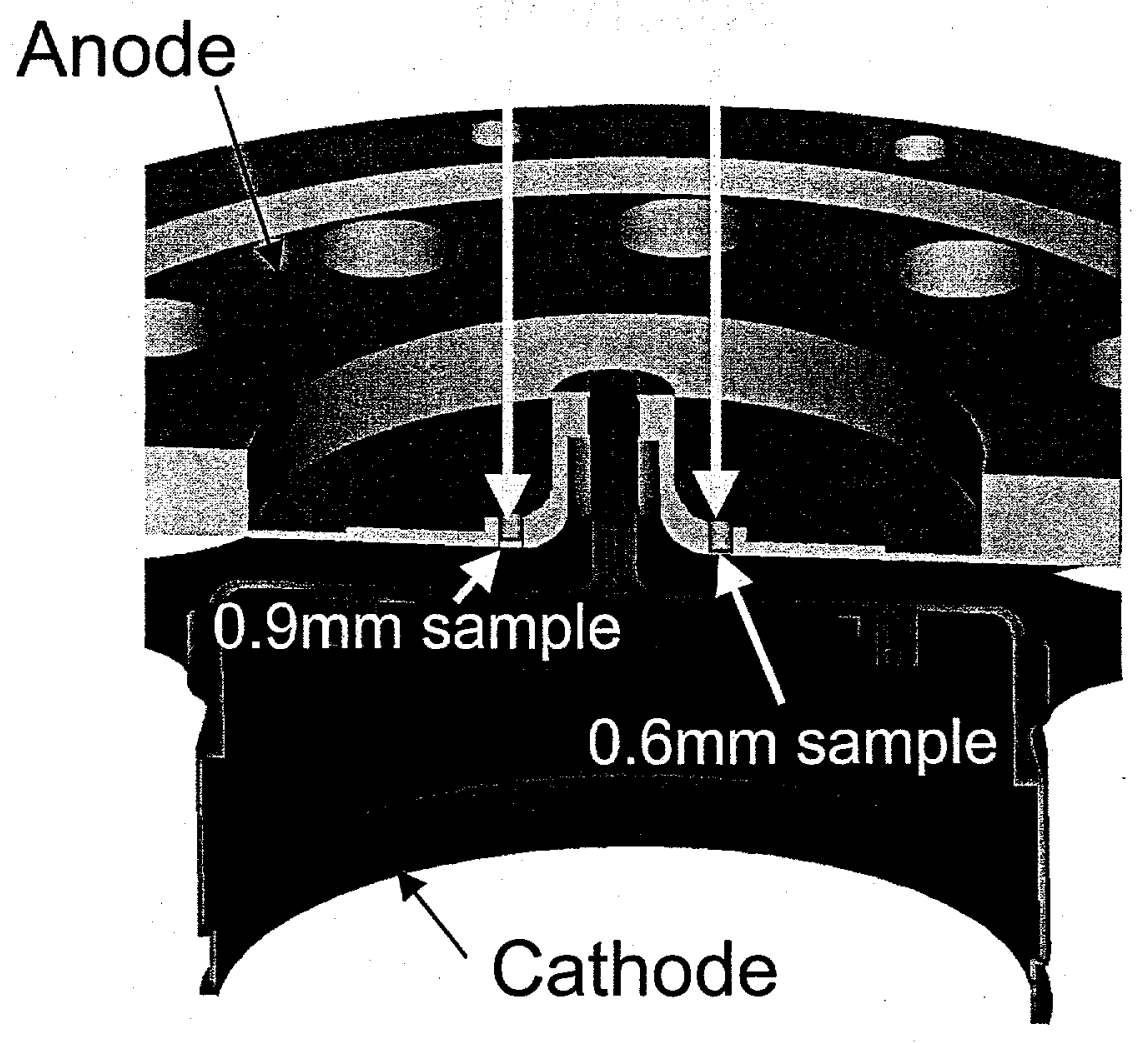

Figure 2a 


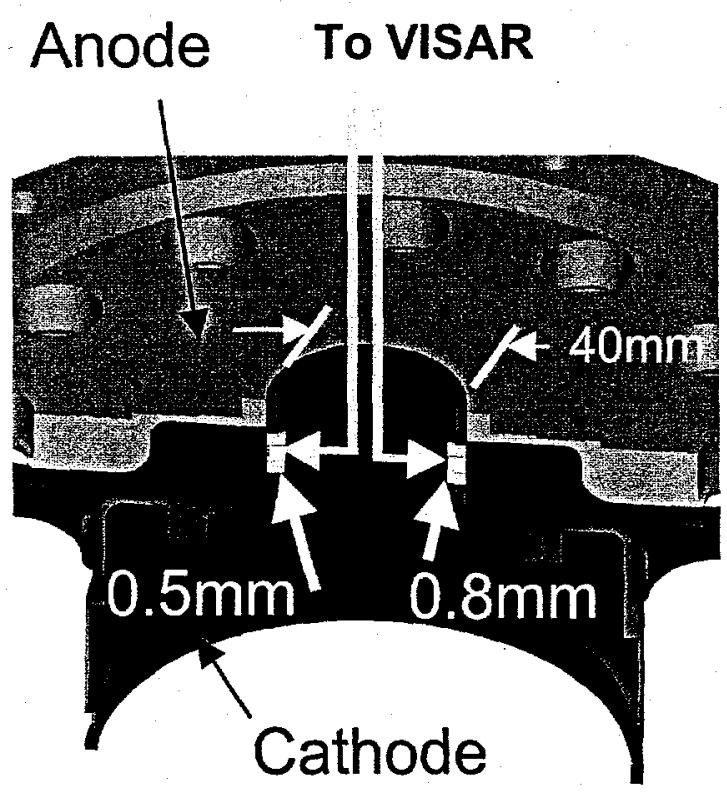

Figure 2b 


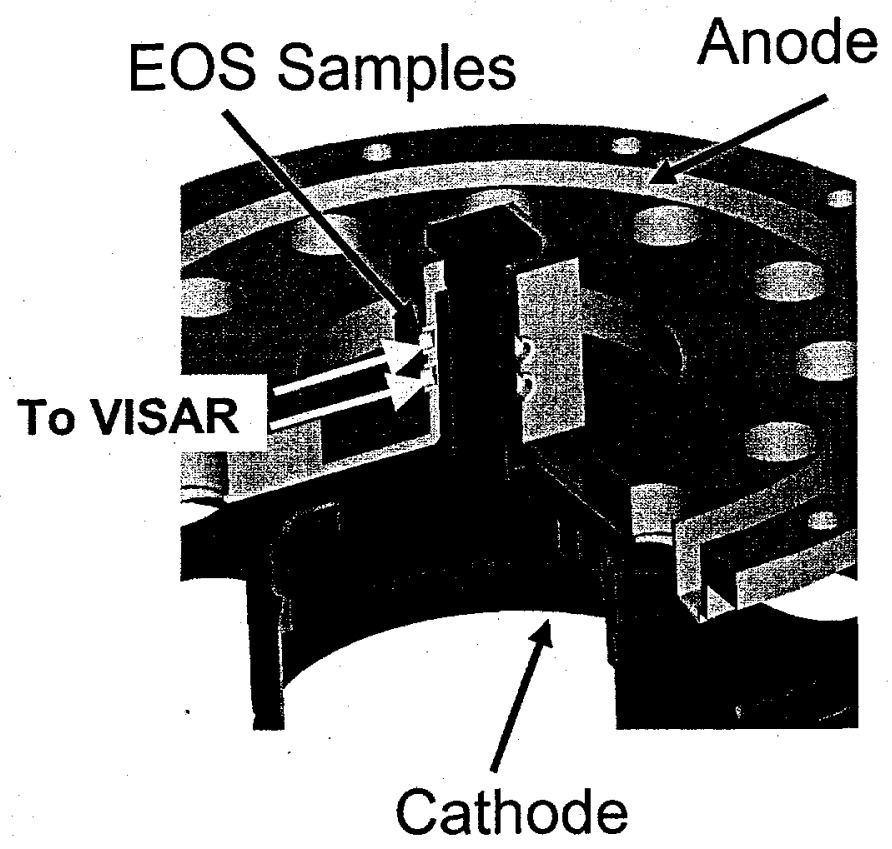

Figure 2c 


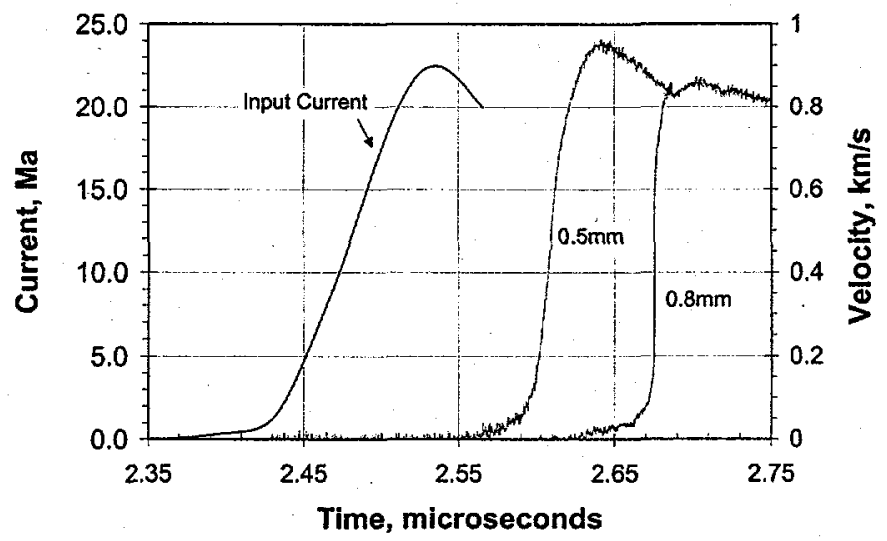

Figure 4 


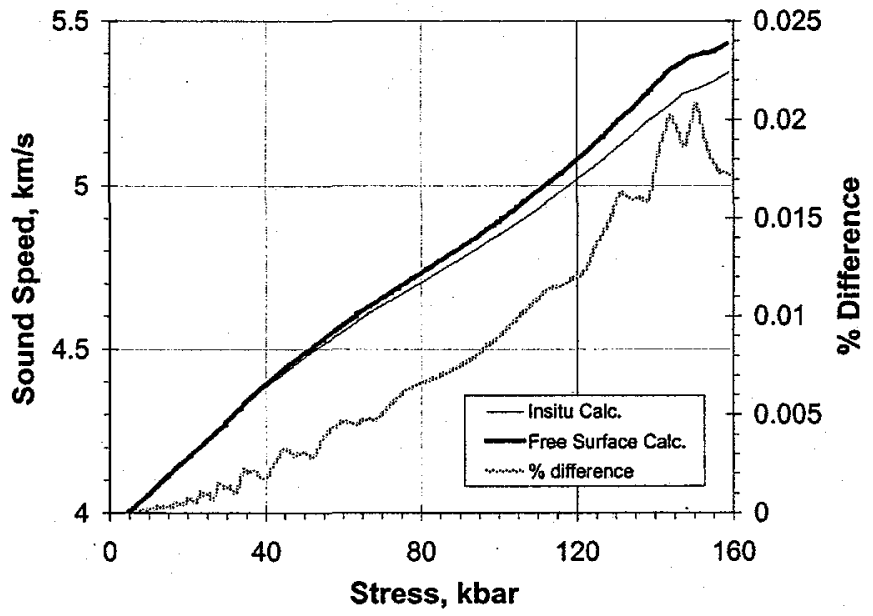

Figure 5 
Z452, Sound speed

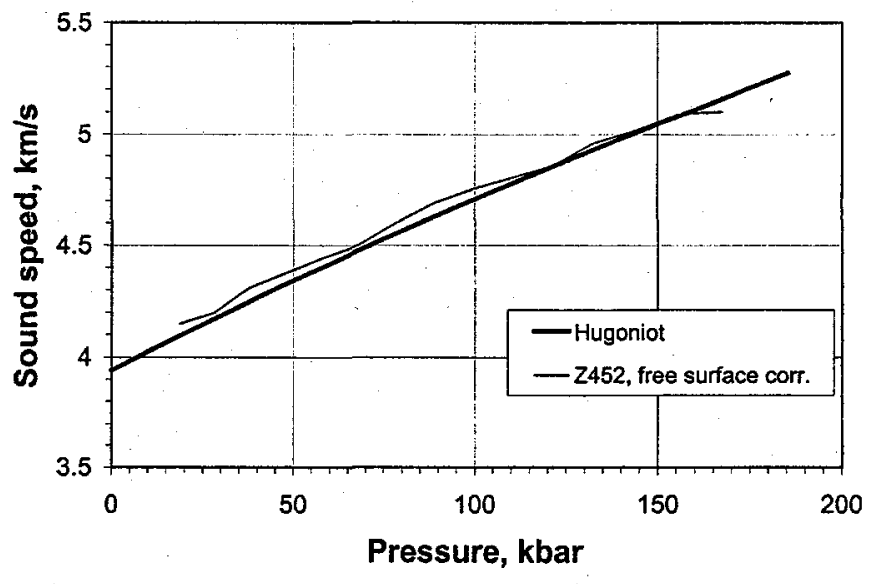

Figure 6 
Hugoniot vs. Isentropic Loading

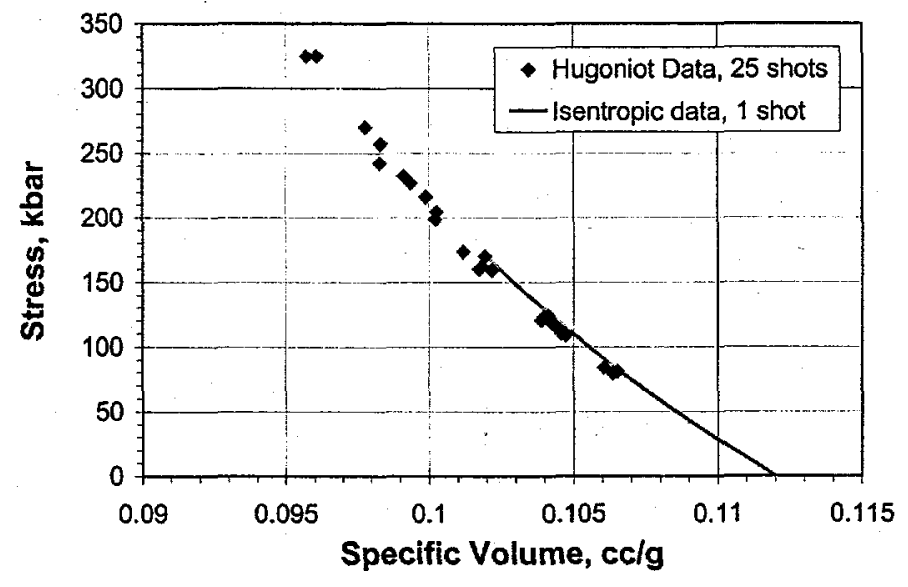

Figure 7 


\section{Shock Loading}

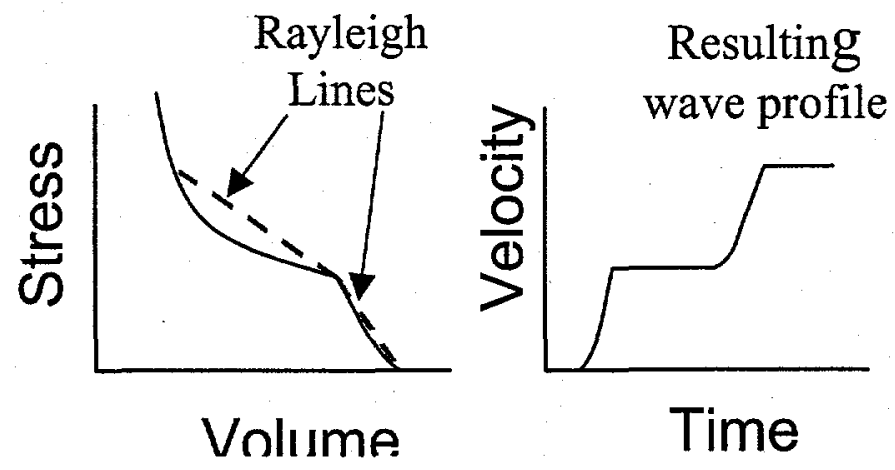

Figure 8 


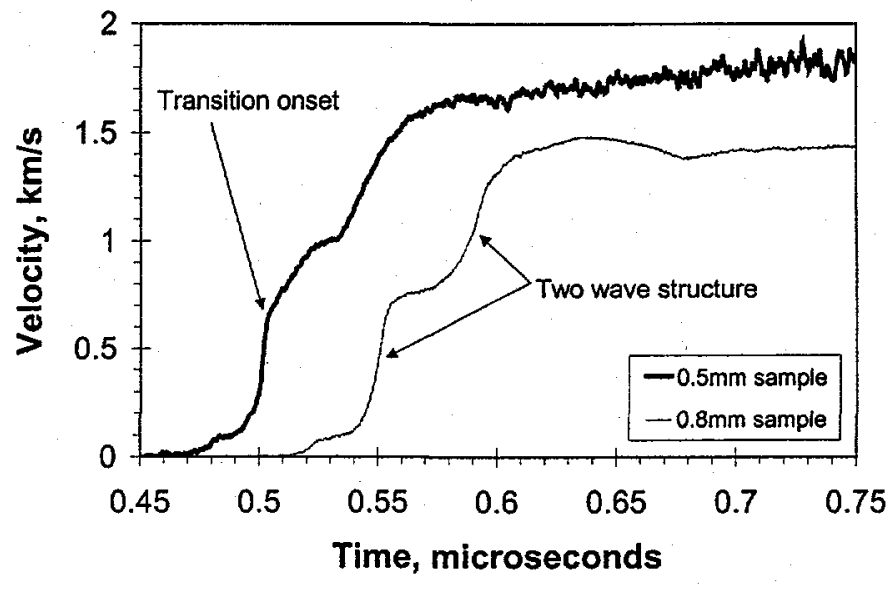

Figure 9 

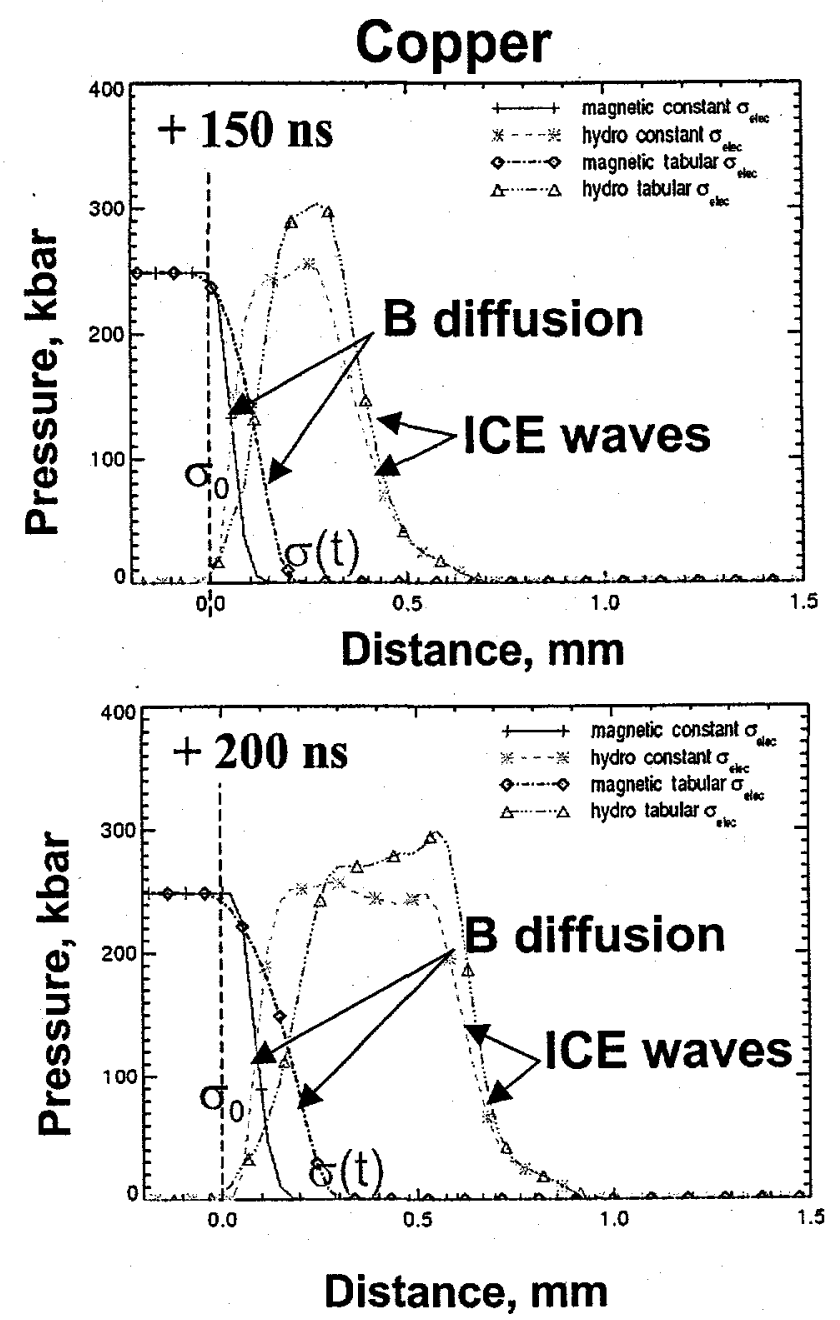

Figure 3 